\title{
Les innovations pédagogiques en enseignement supérieur : pédagogies actives en présentiel et à distance
}

Denis Bédard et Benoît Raucent

\section{(2) OpenEdition \\ Journals}

Édition électronique

URL : http://journals.openedition.org/ripes/898

DOI : 10.4000/ripes.898

ISSN : 2076-8427

Éditeur

Association internationale de pédagogie universitaire

\section{Référence électronique}

Denis Bédard et Benoît Raucent, «Les innovations pédagogiques en enseignement supérieur pédagogies actives en présentiel et à distance », Revue internationale de pédagogie de l'enseignement supérieur [En ligne], 31(1) | 2015, mis en ligne le 23 février 2015, consulté le 22 septembre 2020. URL http://journals.openedition.org/ripes/898 ; DOI : https://doi.org/10.4000/ripes.898

Ce document a été généré automatiquement le 22 septembre 2020.

Article L.111-1 du Code de la propriété intellectuelle. 


\title{
Les innovations pédagogiques en enseignement supérieur : pédagogies actives en présentiel et à distance
}

\author{
Denis Bédard et Benoît Raucent
}

1 En juin 2013, se tenait à l'Université de Sherbrooke (Canada) la septième édition du colloque Questions de pédagogie dans l'enseignement supérieur (QPES). Plus d'une centaine de communications ont été présentées au cours des trois jours qu'a duré cet événement scientifique, qui mettait en avant le thème des innovations pédagogiques dans l'enseignement supérieur. C'est de l'ensemble de ces communications que proviennent les sept textes qui sont présentés dans cette édition spéciale de RIPES.

2 À notre époque, l'innovation pédagogique représente un enjeu clé dans le développement des institutions d'enseignement supérieur à travers le monde. En effet, poussée par des mouvements comme le Scholarship of Teaching and Learning (SoTL) (Boyer, 1990) en Amérique ou par des grandes initiatives politiques comme la déclaration de Bologne, en 1999, en Europe, l'innovation pédagogique représente un des principaux leviers de progrès de toute institution d'enseignement. Sauf à penser que l'enseignement supérieur est proche de la perfection, il nous semble peu utile de poser la question : « Pourquoi innover en enseignement supérieur ? » Il importe plutôt de chercher à définir les contours de ce qu'est l'innovation pédagogique et de ses manifestations dans l'espace de formation du supérieur (Bédard \& Béchard, 2009). Trois enjeux principaux seront mis en évidence ici.

3 Le premier concerne les conditions qui favorisent ou inhibent l'innovation. Tout changement est exigeant pour celles et ceux qui sont concernés : il en va de même pour l'innovation pédagogique. Les personnes qui s'engagent dans une démarche d'innovation doivent donc s'interroger sur l'impact que ce changement est susceptible d'avoir, sur elles-mêmes et sur les autres. Pour faciliter l'acceptation du changement, elles doivent préciser les valeurs et la vision qu'elles proposent et dans lesquelles 
s'inscrit ce changement. Parallèlement, tout projet d'innovation pédagogique doit nécessairement prendre en compte les contextes académique et administratif, voire politique, dans lesquels il prend place. Les actions menées et les mesures prises en considérant chacun de ces contextes sont susceptibles de favoriser ou d'inhiber l'innovation.

4 Un deuxième enjeu à prendre en compte lorsqu'il est question d'innovation pédagogique est celui des zones de tensions qu'elle suscite. Ainsi, celui qui innove est susceptible d'être perçu comme créatif, capable de prendre des initiatives et des risques. Inversement, celui qui n'innove pas peut se sentir exclu ou mis sur la touche en étant confronté aux projets de ses collègues qui cherchent à innover. Que ce soit en lien avec les orientations prises pour la formation des étudiants (par exemple: professionnalisantes vs disciplinaires) ou les stratégies d'enseignement proposées (par exemple : apprentissage par la pratique vs par la transmission des savoirs), des tensions peuvent être présentes dans les milieux de formation où certains bousculent les idées reçues. Ceux qui militent pour le changement et qui pilotent des projets d'innovation doivent prendre en compte l'existence de ces tensions afin de tendre à en diminuer l'impact.

5 Enfin, le troisième et dernier enjeu est celui de la pérennité des innovations pédagogiques mises en place. La littérature sur le sujet montre bien que tout projet d'innovation peut péricliter avec le temps, particulièrement lorsque les acteurs qui portent le changement n'ont pas pris la mesure de ce possible dénouement. En effet, comme dans beaucoup de milieux, le changement se heurte, dans les milieux de formation, à une certaine inertie, en particulier lorsqu'il s'agit de changement pédagogique remettant en cause des pratiques bien établies. Cette résistance au mouvement de changement peut venir des personnes comme des structures. Avec le temps, ce sont elles qui pourront faire faire «marche arrière » au projet d'innovation, parfois progressivement, parfois plus brusquement. Il importe donc d'adopter une vision à long terme du changement afin de voir venir les écueils qui apparaîtront invariablement. C'est ainsi que des compromis et des concessions devront être acceptés en cours de route quant aux actions à prendre, mais ils ne devraient jamais mettre en cause le cœur de l'innovation, c'est-à-dire sa philosophie et ses valeurs. C'est à ce prix que la démarche structurée de changement sera mobilisatrice au départ et pérenne dans le temps.

6 Les trois enjeux évoqués ici s'inscrivent, en toile de fond, dans tout projet d'innovation pédagogique, comme ceux présentés à travers les différents articles de ce numéro spécial. Quatre articles de cette édition spéciale se focalisent sur le changement de posture de l'enseignant et la nécessité d'accompagner ce changement. Ce passage de l'enseignant «traditionnel » à l'enseignant «tuteur » n'est pas nécessairement aisé pour ceux qui doivent prendre le virage de l'APP, par exemple. Il exige de ces personnes qu'elles s'inscrivent dans une posture épistémique particulière vis-à-vis du savoir et qu'elles assument des rôles et des tâches qui ne leur sont pas forcément familiers. Christelle Lison, Denis Bédard et Josée-Anne Côté dans leur article sur le tutorat en apprentissage par problèmes proposent une analyse des styles d'animation adoptés par des tuteurs en médecine et en ingénierie. L'article sur le leadership dans les groupes d'étudiants de Caroline Verzat, Noreen O'Shea et Benoît Raucent met en évidence le fait que les tuteurs s'inscrivent prioritairement dans une logique de régulation de la dynamique de production au détriment de la régulation des dynamiques sociales de 
leadership, alors que cette dernière facette est déterminante pour faciliter la collaboration active en petit groupe. Il est donc important d'aider le tuteur à mener à bien son nouveau rôle. Chantal Morin et Annie Carrier dans leur article le développement de la rétroaction centrée sur l'apprenant proposent de développer la réflexivité chez les enseignants afin de favoriser le passage d'une posture centrée sur le contenu à une posture centrée sur l'étudiant et sa construction de connaissances. Enfin, Louise M. Bélair, Isabelle Vivegnis et Josée Lafrance, dans leur article traitant de l'évaluation de la mise en œuvre d'un dispositif de formation se penchent sur la validation des dispositifs de formation des enseignants à leurs nouveaux rôles.

7 Les trois autres articles proposent des exemples intéressants de dispositifs de pédagogie active mis en œuvre : ils apportent des ouvertures sur l'usage du podcasting et de la vidéo. Nicolas Roland et Philippe Emplit présentent une analyse sur la manière dont les étudiants intègrent le podcasting dans leurs stratégies d'apprentissage au sein d'un dispositif donné. Dans leur article sur la transformation des conceptions naïves, Benoît Raucent, Christian Ritter, Jim Plumat, Pascale Corten-Gualtieri et Marcel Lebrun abordent la problématique des conceptions naïves en physique qui sont portées par les étudiants à leur entrée à l'université et l'utilisation de vidéos comme outils de questionnement et d'apprentissage. Enfin, Céline Weyermann, Amaury Daele, Cyril Muehlethaler et Romain Voisard soulèvent la question de la motivation des étudiants.

Au-delà des différentes thématiques abordées, les articles de ce numéro spécial témoignent que l'innovation devrait être un projet et non seulement un idéal. L'idéal on y rêve, le projet on le réalise.

Bonne lecture!

\section{AUTEURS}

\section{DENIS BÉDARD}

Université de Sherbrooke

2500 boul. Université, Sherbrooke, Québec, Canada

denis.bedard@usherbrooke.ca

\section{BENOÎT RAUCENT}

Université Catholique de Louvain

IPM, Louvain-la-Neuve, Belgique

benoit.raucent@uclouvain.be 